\title{
Automedicação em idosos e fatores associados
}

\author{
Self-medication in elderly and associated factors \\ Automedicación en ancianos y factores associados
}

\begin{abstract}
Ezequiel Cássio Gusmão시 Lorena Aguilar Xavier ${ }^{2 *}$, Gabriel Alencar Mota ${ }^{2}$, Ítalo Augusto Araújo de Deus $^{2}$, Laila Tamires Gomes Santana², Débora Mayra de Freitas Veloso², Marina Ramos Costa ${ }^{3}$, Lanuza Borges Oliveira ${ }^{2,4}$, João Marcus Oliveira Andrade ${ }^{2}$, Igor Dorze de Alencar e Castro ${ }^{3}$, Karina Andrade de Prince ${ }^{4}$, Marcos Vinicius Macedo de Oliveira², Luçandra Ramos Espirito Santo ${ }^{1,2,4}$.
\end{abstract}

\section{RESUMO}

Objetivo: Identificar fatores associados à prática da automedicação em idosos da cidade de Montes Claros/Minas Gerais/Brasil. Métodos: Este estudo tem desenho transversal, descritivo, realizado com idosos entrevistados em drogaria em Montes Claros. Realizaram-se 302 entrevistas coletando aleatoriamente dados como idade, escolaridade, fármacos estocados, condições de armazenamento e frequência da utilização. As informações coletadas foram transferidas para um banco de dados do software Statistical Package for the Social Sciences - SPSS, através do qual foram avaliadas possíveis relações de associação entre as variáveis. Foi utilizado o teste qui-quadrado para investigar a existência de associações entre as variáveis independentes. Resultados: $92,4 \%$ realizam automedicação e $97,66 \%$ deles faz uso de algum medicamento diariamente. Houve um predomínio da automedicação na faixa etária de 60 até 66 anos $(28,8 \%)$ e menor concentração entre 66 e 69 anos (22,8\%). As medicações de maior utilização foram os anti-hipertensivos e anti-inflamatórios. Conclusão: Apesar de risco à saúde, a automedicação ocorre frequentemente entre idosos. Este estudo vem enfatizar a necessidade de promoção do uso racional de medicamentos neste segmento populacional.

Palavras-Chaves: Automedicação, Uso de Medicamentos, Saúde do Idoso.

\begin{abstract}
Objective: To identify factors associated with the practice of self-medication among the elderly in the city of Montes Claros / Minas Gerais / Brazil. Methods: This cross - sectional, descriptive study was performed with elderly individuals interviewed in a drugstore in Montes Claros. A total of 302 interviews were conducted randomly collecting data such as age, schooling, drugs stored, storage conditions and frequency of use. The information collected was transferred to a database of the Statistical Package for the Social Sciences (SPSS), through which possible associations between the variables were evaluated. The chi-square test was used to investigate the existence of associations between the independent variables. Results: $92.4 \%$ self-medicate and $97.66 \%$ use some medication daily. There was a predominance of self-medication in the age group of 60 to 66 years $(28,8 \%)$ and a lower concentration between 66 and 69 years $(22,8 \%)$. The most commonly used medications were antihypertensives and anti-inflammatories. Conclusion: Despite health risk, self-medication often occurs among the elderly. This study emphasizes the need to promote the rational use of drugs in this population segment.
\end{abstract}

Keywords: Self Medication, Drug Utilization, Health of the Elderly.

\footnotetext{
${ }^{1}$ Faculdades Unidas do Norte de Minas (FUNORTE), Montes Claros-MG.

2 Universidade Estadual de Montes Claros-MG (UNIMONTES). ${ }^{*}$ E-mail: lorenaaguilarx@gmail.com

${ }^{3}$ Universidade Federal do Estado do Rio de Janeiro (UNIRIO).

${ }^{4}$ Faculdades Integradas Pitágoras (FIPMOC), Montes Claros-MG.
} 


\section{RESUMEN}

Objetivo: Para identificar factores asociados con la práctica de la auto-meditación entre los ancianos en la ciudad de Montes Claros / Minas Gerais / Brazil. Métodos: Este estudio tiene un diseño transversal, descriptivo, realizado con ancianos entrevistados en droguería en Montes Claros. Se realizaron 302 entrevistas recogiendo aleatoriamente datos como edad, escolaridad, fármacos almacenados, condiciones de almacenamiento y frecuencia del uso. La información recogida fue transferida a una base de datos del software Statistical Package for the Social Sciences - SPSS, a través del cual se evaluaron posibles relaciones de asociación entre las variables. Se utilizó la prueba chi-cuadrada para investigar la existencia de asociaciones entre las variables independientes. Resultados: $92,4 \%$ realizan automedicación y el 97,66\% de ellos hace uso de algún medicamento diariamente. Se observó un predominio de la automedicación en el grupo de edad de 60 a 66 años (28,8\%) y menor concentración entre 66 y 69 años (22,8\%). Los medicamentos de mayor uso fueron los antihipertensivos y antiinflamatorios. Conclusión: A pesar de riesgo a la salud, la automedicación ocurre frecuentemente entre ancianos. Este estudio viene a enfatizar la necesidad de promover el uso racional de medicamentos en este segmento poblacional.

Palavras-Clave: Automedicación, Utilización de Medicamentos, Salud del Anciano.

\section{INTRODUÇÃO}

Automedicação é o consumo de medicamentos sem prescrição médica, em que o paciente decide sozinho qual medicamento utilizar. Trata-se de um fenômeno potencialmente nocivo à saúde, uma vez que nenhum medicamento é inócuo ao organismo. O uso indevido de medicamentos considerados "inofensivos" pode acarretar consequências como resistência bacteriana, reações de hipersensibilidade, dependência, sangramento digestivo, sintomas de retirada, bem como podem aumentar o risco para neoplasias, hemorragia cerebral devido à combinação de um anticoagulante com um simples analgésico, por exemplo. Além disso, 0 alívio momentâneo dos sintomas pode mascarar a doença de base que passa despercebida e que pode, assim, progredir (WHO, 1998; BARROSO et al.,2017).

No Brasil, onde o acesso à assistência médica ainda encontra dificuldades em ser universal, em que 0 Sistema Público de Saúde não tem condições de atender a população com a agilidade e a qualidade que deveriam, aliado a uma grande parcela da sociedade na faixa da pobreza que não tem condições financeiras para pagar pelos serviços de saúde, a prática da automedicação torna-se bastante comum. Todavia, somente o fator financeiro não é suficiente para explicar a prática da automedicação. Fatores como escolaridade, classe social, acesso às informações a respeito dos medicamentos e, inclusive, o fator cultural também exercem interferência nesse aspecto (SOUZA, 2008; BARROSO et al.,2017).

O crescimento contínuo do consumo de medicamentos entre os idosos pode ser justificado pelo aumento da prevalência de doenças crônicas nessa faixa etária, bem como ao modelo de saúde que tem no medicamento sua principal forma de intervenção. No entanto, as implicações desse consumo precisam ser medidas e avaliadas quanto ao seu risco/benefício (COSTA \& PEDROSO, 2011).

Associado à automedicação está o uso comum de uma farmácia doméstica representada na estocagem de medicamentos nas residências. Esse estoque doméstico apresenta vários riscos, entre eles há o problema intrínseco da facilidade de acesso à medicamentos sem orientação médica e as condições de armazenamento, que podem comprometer a eficácia do fármaco. Os medicamentos devem ser guardados em local arejado e seguro, sem exposição à luz, calor ou umidade, em sua embalagem original, identificados pelo nome comercial ou genérico e princípio ativo e com data de validade e lote, o que nem sempre ocorre (MASTROIANNI et al., 2011).

Os idosos possuem maior vulnerabilidade à automedicação, uma vez que os mesmos consomem mais medicamentos que outros grupos etários, uma média diária de dois a cinco medicamentos por dia, sendo mais suscetíveis a ocorrência de iatrogenia, além de apresentarem comprometimento no metabolismo hepático e na depuração renal. Além disso, nota-se que o consumo de medicamentos quase triplica com o 
avançar da idade sendo tal fato justificado pela menor tolerância frente a sintomas agudos como por exemplo, a dor determinando uma maior necessidade por fármacos especialmente quanto maior for o grau de automedicação nesses indivíduos. Dessa forma, eles se tornam mais propensos ao uso indiscriminado e aos eventos adversos responsáveis pelo aumento da internação hospitalar e óbito nesse grupo de indivíduos. Assim sendo, o hábito da automedicação é algo que ocorre com frequência significativa em nosso país, sendo uma prática considerada como um grave problema de saúde pública. (PEREIRA et al.,2017).

Neste sentido, este estudo teve como objetivo o conhecimento acerca da automedicação e possíveis intoxicações medicamentosas em uma população de idosos.

\section{MÉTODOS}

O presente trabalho trata-se de estudo transversal, descritivo, quantitativo, retrospectivo. Foi realizado com uma população de adultos, na sua maioria composta por idosos, atendidos em drogaria na cidade de Montes Claros que aceitaram participar da pesquisa. A cidade de Montes Claros está localizada ao norte do estado de Minas Gerais, apresenta uma população de 361915 habitantes, segundo dados do IBGE obtidos no último censo realizado em 2010.

O estudo ocorreu no período compreendido entre Agosto de 2016 a Julho de 2018. Foi utilizado como instrumento de pesquisa uma entrevista estruturada com dados para coleta da variável dependente automedicação e variáveis independentes como idade, escolaridade, estado civil, uso diário de medicação, condições de armazenamento, descarte dos medicamentos e conferência da validade dos medicamentos. Foram realizadas um total de 302 entrevistas que aceitaram participar da pesquisa que compareciam no estabelecimento da pesquisa. A coleta de dados ocorreu de forma aleatória, em ambiente arejado, onde foi mantido o sigilo e confidencialidade dos dados na entrevista, procurando deixar o participante em posição confortável, de preferência sentado, para responder às perguntas elencadas.

As informações coletadas foram codificadas e transferidas para um banco de dados do software analítico Statistical Package for the Social Sciences - SPSS, versão 23.0, (SPSS for Windows, Chicago, EUA), através do qual foram avaliadas possíveis relações de associação entre as variáveis. Foi o utilizado o teste do quiquadrado para investigar a existência de associações entre as variáveis independentes. Para todas as análises foi considerado um nível de significância $p<0.05$.

O presente estudo obedeceu aos princípios éticos definidos pelo Conselho Nacional de Saúde através da resolução 466/12 para realização de pesquisa em seres humanos. Todos os sujeitos abordados foram inicialmente informados quanto à identificação dos entrevistadores, os objetivos da pesquisa, da não obrigatoriedade de participação e a possibilidade de abandono a qualquer momento. Todos os participantes desse estudo assinaram o termo de consentimento livre e esclarecido. O projeto apresenta parecer favorável número 1.571.501/2016 pelo comitê de ética, além da aprovação pelo Conselho de Ensino, pesquisa e Extensão- Resolução 132-CEPEx Unimontes.

\section{RESULTADOS}

A população estudada foi composta por indivíduos de 60 a 88 anos de idade, com maior predomínio daqueles entre 60 e 66 anos que representaram $28,8 \%$ dos entrevistados e uma menor percentagem dos idosos entre 74 e 88 anos, os quais equivalem apenas $22,5 \%$ da amostra. Quanto ao estado civil a maior representatividade era das pessoas casadas, o que equivaleu a $61,6 \%$ dos entrevistados e a parcela de viúvos era $28,5 \%$. No quesito grau de instrução houve um predomínio de indivíduos com o ensino médio completo o que caracterizou $41,06 \%$ da amostra e que não trabalhavam, os quais equivalem a $81,13 \%$, condizente com a idade avançada onde se encontram os aposentados.

Os resultados demonstraram que a automedicação é um problema de saúde publica comum entre os idosos. Dentre os entrevistados, a maioria $278(92,4 \%)$ respondeu que adquire medicamentos sem prescrição 
médica, sendo que quase a totalidade dos analisados $(97,66 \%)$ faz uso de algum medicamento diariamente e apenas $3,01 \%$ relatou que alguém acompanha e monitora a dose do medicamento. $65 \%$ das pessoas que afirmaram se automedicar eram mulheres e $35 \%$ homens.

Quanto ao armazenamento e descarte dos medicamentos observou-se um predomínio do armazenamento na cozinha e quarto, $42,19 \%$ e $46,84 \%$ respectivamente e o maior índice de descarte foi no lixo comum, a grande maioria $83,61 \%$ sendo que o restante referiu o vaso sanitário ou outro local para descarte dos medicamentos.

Em relação ao perfil dos idosos que se automedicam nota-se um predomínio na faixa etária de 60 até 66 anos (28,8\%) e menor concentração entre 66 e 69 anos (22,8\%) e entre 74 e 88 anos (22,5\%), porém não houve significância estatística $(p>0,01)$ evidenciando que a idade e a automedicação são independentes.

Tabela 1: Distribuição das características sociodemográficas e demais variáveis correlacionando a automedicação em idosos como variável dependente.

\begin{tabular}{|c|c|c|c|}
\hline VARIÁVEL & $\mathbf{N}$ & $\%$ & $p$ valor \\
\hline \multicolumn{4}{|l|}{ Idade } \\
\hline $60-66$ anos & 87 & 28,8 & \multirow{6}{*}{0,150} \\
\hline $66-69$ anos & 69 & 22,8 & \\
\hline $69-74$ anos & 71 & 23,5 & \\
\hline $74-88$ anos & 68 & 22,5 & \\
\hline Não informado & 7 & 2,3 & \\
\hline Total & 302 & 100 & \\
\hline \multicolumn{4}{|l|}{ Estado civil } \\
\hline Solteiro & 1 & 0,3 & \multirow{6}{*}{0,688} \\
\hline Casado & 186 & 61,6 & \\
\hline Viúvo & 86 & 28,5 & \\
\hline Sem resposta & 4 & 1,3 & \\
\hline Outro & 25 & 8,3 & \\
\hline Total & 302 & 100 & \\
\hline \multicolumn{4}{|l|}{ Escolaridade } \\
\hline Sem superior & 244 & 80,8 & \multirow{3}{*}{$<0,001$} \\
\hline Com superior & 58 & 19,2 & \\
\hline Total & 302 & 100 & \\
\hline \multicolumn{4}{|c|}{ Análise da validade dos medicamentos } \\
\hline $\operatorname{Sim}$ & 107 & 35,4 & \multirow{4}{*}{$<0,001$} \\
\hline Não & 191 & 63,2 & \\
\hline Não informaram & 4 & 1,3 & \\
\hline Total & 302 & 100 & \\
\hline
\end{tabular}

Fonte: Elaborado pelos Autores

REAS/EJCH | Vol. 11 (2) | e191 | DOI: https://doi.org/10.25248/reas.e191.2019 Página 4 de 8 
Dentre os indivíduos que relataram comprar medicamentos sem prescrição médica $28,5 \%$ são viúvos enquanto que $61,6 \%$ são casados, porém também não houve significância estatística ( $p>0,01)$.

Dentre os indivíduos que responderam que compram medicação sem prescrição médica $80,8 \%$ não possuem ensino superior com significância estatística $(p<0,01)$. (Tabela 1)

Apesar do alto índice de idosos que alegam se automedicar apenas 6,2\% afirmam ter sofrido algum episódio de intoxicação e destes apenas $0,33 \%$ necessitou de internação hospitalar em decorrência do quadro. Quanto aos cuidados referentes à análise da validade dos medicamentos $63,2 \%$ dos indivíduos que referiram se automedicar não tem o hábito de conferir a validade dos medicamentos que faz uso com nível de significância menor que 0,01. (Tabela 1)

Com relação a qual medicamento mais comum entre as medicações usadas, os anti-hipertensivos e antiinflamatórios formam as classes terapêuticas mais utilizadas.

\section{DISCUSSÃO}

Este trabalho vem demonstrar a alta prevalência da automedicação entre os idosos de Montes Claros, além de destacar algumas características que podem estar, em maior ou menor grau, relacionadas à maior prevalência do fenômeno. A automedicação é um fator de risco para a saúde, especialmente em idosos, e este fator deve ser levado em conta quando se tratando de comorbidades que podem estar relacionadas ao abuso ou uso incorreto de medicamentos, como as intoxicações ou resistência bacteriana.

Corroborando com os resultados encontrados nesse estudo, outro estudo mostra que a terceira idade é uma fase da vida caracterizada por uma maior propensão ao desenvolvimento de comorbidades agudas e crônicas, em diversos sistemas do organismo, como esquelético, cardiovascular e metabólico; e, portanto, também é uma fase com maior propensão à necessidade da utilização temporária e ou contínua de fármacos (ARAÚJO e GALATO, 2012).

Quanto ao fato da compra de medicamentos sem prescrição médica entre os entrevistados foi observado que $92,4 \%$ recorre a essa prática, o que demonstra bem a frequência do hábito entre sujeitos idosos, na cidade de Montes Claros. Este resultado é ratificado também por diversos outros estudos realizados em nosso país, como os estudos de Sá et al. (2007), Monteiro et al. (2014) e Telles Filho et al. (2013), que demonstraram prevalência nas porcentagens de $77,2 \%, 67 \%$ e $100 \%$, respectivamente, demonstrando que este é um hábito arraigado à cultura brasileira.

Em um estudo realizado em idosos no Rio de Janeiro (TELLES FILHO et al., 2013), quando os sujeitos foram questionados quanto à frequência com que recorreram à automedicação no ano anterior, $88 \%$ afirmaram ter recorrido mais de 10 vezes ao mês e $12 \%$ menos de 2 vezes, fato também preocupante, pois os idosos apresentam alterações fisiológicas que os tornam mais propensos às reações adversas dos medicamentos. Tal situação é similar aos resultados encontrados em que mais de $90 \%$ dos participantes recorreram à automedicação.

Em outros estudos também foi demonstrado que a automedicação entre idosos estava majoritariamente presente entre as mulheres, com a ressalva de que as pesquisas envolviam mulheres em sua maioria, devido à maior longevidade do sexo feminino. Essa tendência seria explicada por diversas questões, como: o maior autocuidado, a representação do papel de cuidadora e protetora da família, e as visitas mais frequentes às farmácias e aos serviços de saúde (TELLES FILHO et al., 2013; PEREIRA et al.,2017).

Além disso, é relevante destacar que no presente estudo uma porcentagem ínfima $(3,01 \%)$ dos entrevistados que utilizavam medicamentos diariamente relatou acompanhar e monitorar a dose dos medicamentos. Junto à conjuntura do problema, isto poderia leva-los também a, no momento da compra do fármaco sem a receita, errar a dose prescrita; ou ainda, pela falta de acompanhamento, continuar sempre comprando e utilizando os medicamentos numa mesma dose, mesmo que esteja defasada e necessite ser corrigida, como no caso de alteração da prescrição pelo médico. Cabe ressaltar a importância do profissional farmacêutico na dispensação correta dos medicamentos, afim de orientar o uso correto das fármacos. 
A maioria dos idosos $(67,5 \%)$ relatou ainda não ter o hábito de conferir a validade dos medicamentos, medida esta que, além de necessária para o correto controle dos medicamentos utilizados e adquiridos, se faz ainda mais notória no contexto da utilização de medicações em longo prazo, como é o caso dessa população estudada, que acabam, portanto, por armazenar grandes quantidades de medicamentos, por longos períodos.

Quanto ao descarte dos medicamentos, corroborando os nossos resultados outros estudos apontam que as repercussões negativas do descarte inadequado estão relacionadas a um grande impacto ambiental determinado pela contaminação do solo, águas, rios, lagos, oceanos, águas subterrâneas e lençóis freáticos. Tais consequências são resultado do contato de catadores com os medicamentos que são descartados juntamente aos resíduos comuns fazendo com que os trabalhadores tenham contato com esses materiais descartando-os diretamente no solo para que o reaproveitamento das embalagens seja possível. Além disso, é bastante prevalente a eliminação de medicamentos isolados ou em recipientes na rede pública de esgoto, sendo um grande desafio para as empresas de saneamento responsáveis pelo tratamento do esgoto fazer o manejo dessas águas com a presença de fármacos (PINTO et al., 2014; BILA; DEZOTTI, 2003; ZAPARROLI et al., 2011).

Entre os estudos, diversas são explicações levantadas para o fenômeno da automedicação, como: a baixa conscientização sobre os riscos do hábito, a dificuldade de acesso a serviços de saúde e as experiências positivas prévias com o uso dos medicamentos (MONTEIRO et al., 2014).

No entanto, é visto também que há influência cultural e social no contexto. No estudo realizado no Rio de Janeiro, os sujeitos, quando questionados quanto à justificativa da utilização dos medicamentos, apenas 10\% relataram ser devido à dificuldade de acesso ao atendimento médico. As outras justificativas foram: ter o medicamento em casa $(58 \%)$, facilidade para comprar (10\%), indicação familiar $(8 \%)$, experiência prévia positiva (8\%) e não ter julgado necessária a consulta médica (6\%). Entretanto, os mesmos sujeitos, quando indagados sobre qual seria a principal influência para que realizassem a automedicação, $62 \%$ referiu ser a publicidade / mídia (televisão, revistas), 36\% relacionou à influência de familiares e amigos e $2 \%$ aos profissionais de saúde (não-médicos) (TELLES FILHO et al., 2013). Estes dados mostram que, na gênese do problema, há de se considerar também os fatores de cunho social, e não apenas o grau de instrução e a facilidade de acesso aos serviços de saúde.

Vale ressaltar que entre os medicamentos mais frequentemente mencionados na automedicação, seja neste ou em outros estudos, estão os anti-inflamatórios e analgésicos. Nesse contexto, estudos nacionais e internacionais também têm demonstrado que a prática em questão estaria mais frequentemente associado à presença de sinais e sintomas de característica aguda, como dor e febre, sintomas comuns nessa fase da vida (SÁ et al., 2007 e MONTEIRO et al., 2014).

Esses medicamentos, portanto, ganham relevância no contexto da automedicação, uma vez que, apesar de úteis, apresentam risco para esse grupo populacional, principalmente em se tratando do uso recorrente ou prolongado.

O uso dos anti-inflamatórios não esteroidais (AINES), por exemplo, muito utilizados pela população e na prática clínica, apresenta riscos que o público leigo, desavisado, se expõe pelo próprio uso rotineiro. Dentre os principais efeitos prejudiciais está a irritação da mucosa gástrica, decorrente da inibição não-seletiva das ciclo-oxigenases, enzimas responsáveis pela produção de prostanglandinas cuja função, dentre outras, é a proteção da parede gástrica dos efeitos corrosivos do próprio ácido clorídrico estomacal. Esses medicamentos, quando utilizados em jejum, ou por longos períodos sem a associações de um outro medicamento mucoprotetor, podem causar lesões que tendem a evoluir desde a erosão à perfuração e hemorragia gástricas (BATLOUNI, 2010).

Além disso, estes medicamentos possuem um significativo número de interações medicamentosas, especialmente para o grupo etário, podendo potencializar a ação de anticoagulantes orais, como o clopidogrel, e reduzir a eficácia de anti-hipertensivos como os diuréticos e os inibidores da enzima de conversão da angiotensina I (IECAs) (BATLOUNI, 2010). 
Os próprios analgésicos mais comuns, como a dipirona, também apresentam riscos, como o de intoxicação aguda e crônica, a depender do uso de doses descontroladas, podendo levar a taquipneia, sedação e hemossiderose em fígado e baço. Ademais, possui contraindicações comumente inobservadas, como alterações em medula óssea, gravidez e lactação (BARROSO et al.,2017).

O relatório do Sistema Nacional de Informações Tóxico-Farmacológicas (SINITOX) de 2012 demonstra que aproximadamente $30 \%$ dos casos (27.008) de intoxicações registrados são resultado do uso de medicação sendo 826 destes relacionados à automedicação. (BARROSO et al.,2017)

Esses medicamentos, portanto, podem predispor à maior fragilização do idoso. Não obstante, o uso regular medicamentos, seja para correção de afecções agudas ou crônicas, requer avaliação constante dos riscos à saúde, especialmente no que tange à intoxicações e interações medicamentosas. A prescrição correta deve considerar o risco e benefício das drogas escolhidas, assim como a margem de segurança descrita na literatura, principalmente nas faixas etárias da pediatria e geriatria. Atualmente, o uso irregular de medicamentos ainda é uma das principais causas de intoxicação em nosso país (ARAÚJO e GALATO, 2012). Entende-se que apesar da prática de automedicação poder ser vista por alguns indivíduos como uma forma de autocuidado, já que está relacionada a busca pela manutenção da saúde, prevenção de enfermidades e tratamento de doenças, sua aplicação por não dispor do adequado acompanhamento e orientação por profissionais habilitados culmina com maior riscos do que benefícios aos praticantes. (PEREIRA et al.,2017)

\section{CONCLUSÃO}

Apesar de ser um risco comprovado a saúde, a automedicação ainda permanece como uma prática rotineira pela maioria dos idosos. O uso regular de medicamentos requer avaliação constante dos riscos à saúde, especialmente no que tange às intoxicações, doenças secundárias e interações medicamentosas. A atenção na prescrição e dispensação de medicamentos é algo relevante para a saúde pública, e em particular para os idosos, devido à maior utilização de medicamentos por esses indivíduos e, por este grupo representar parcela considerável da população, uma vez que tem aumentado a expectativa de vida da população brasileira. Diante da importância do tema, esse estudo vem descrever acerca do perfil de uma população de idosos que tem um hábito de se automedicarem. Os resultados apresentados apontam para a necessidade da promoção do uso racional de medicamentos neste segmento populacional, contemplando a promoção de saúde e prevenção de intoxicações nos estabelecimentos privados e públicos, afim de reduzir a morbimortalidade pelo uso incorreto dos medicamentos.

\section{AGRADECIMENTOS}

À Fapemig e Unimontes pelo incentivo à iniciação científica (PROINIC), tanto à concessão de bolsas, quanto ao programa voluntário

\section{REFERÊNCIAS}

1. ARAÚJO PL, GALATO D. Risco de fragilização e uso de medicamentos em idosos residentes em uma localidade do sul de Santa Catarina. Revista Brasileira de Geriatria e Gerontologia, 2012; 15(1): 119-126.

2. BARROSO R, TELLES FILHO PCP, PINHEIRO MLP et al. Automedicação em Idosos de Estratégias de Saúde da Família, Rev enferm UFPE on line, 2017; 11(2):890-7.

3. BATLOUNI M. Anti-inflamatórios não esteroides: Efeitos cardiovasculares, cérebro-vasculares e renais. Arquivos Brasileiros de Cardiologia, 2010; 94(4): 556-563.

4. BILA DM, DEZOTTI M. Fármacos no meio ambiente. Química Nova, 2003; 26(4): 523-530.

5. BORGES RM, MINILLO A, LEMOS EGM et al. Uso de filtros de carvão ativado granular associado a microrganismos para remoção de fármacos no tratamento de água de abastecimento. Revista Engenharia Sanitária e Ambiental, 2016; 21: 1-13. 
6. BRASIL, IBGE. Censo Demográfico, 2010

7. COSTA SC, PEDROSO ERP. A prescrição de medicamentos para idosos internados em serviço de clínica médica: atualização. Revista Medica de Minas Gerais. 2011; 21(2):201-14.

8. FREITAS K. Prevalência de Automedicação na população do município de Fraiburgo (SC). Revista Interdisciplinar de Estudos em Saúde, 2012; 1(1): 38-58.

9. PEREIRA FGF, ARAÚJO MJP, PEREIRA CR et al. Automedicação em Idodos Ativos, , Rev enferm UFPE on line, 2017; 11(12):4919-28

10. SÁ MB, BARROS JAC, SÁ MPBO. Automedicação em idosos na cidade de Salgueiro-PE. Revista Brasileira de Epidemiologia, 2007; 10(1): 75-85.

11. SOUZA HWO, SILVA JL, NETO MS. A importância do profissional farmacêutico no combate à automedicação no Brasil. Revista Eletrônica de Farmácia, 2008; 5(1): 67-72.

12. TELLES FILHO PCP, ALMEIDA AGP, PINHEIRO, MLP. Automedicação em idosos: Um problema de Saúde Pública. Revista Enfermagem, 2013; 21(2): 97-201.

13. WORLD HEALTH ORGANIZATION. The role of the pharmacist in self medication and self-care. International Journal of Clinical Pharmacy, 2014; 36(2): 412-419.

14. ZAPARROLI ID, CAMARA MRG, BECK C. Medidas mitigadoras para a indústria de fármacos Comarca de Londrina-PR. Brasil: impacto ambiental do despejo de resíduos em corpos hídricos. In: $3^{\circ}$ International Workshop Advanced in Cleaner Production. Cleaner Production Initiatives and Challenges for a Sustainable World. São Paulo, 2011. 